\title{
Correction to: Development of a Laser Pistonphone System to Calibrate the Sensitivity Modulus and Phase of Microphones for Infrasonic Frequencies
}

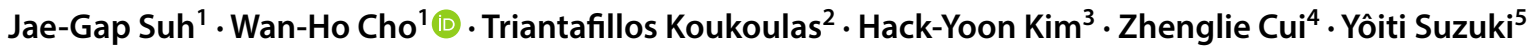

Published online: 20 August 2020

(c) Korean Society for Precision Engineering 2020

\section{Correction to: \\ International Journal of Precision Engineering and \\ Manufacturing (2020) 21:1279-1289 \\ https://doi.org/10.1007/s12541-020-00338-4}

The 3rd author, Triantafillos Koukoulas had changed his affiliation in March. The detail of new address was not firmly decided at the period for submitting the proof of manuscript and the address in the current form is no longer valid.
The new affiliation is:

2 National Research Council Canada, 1200 Montreal Road, Building M36 - Office 1016, Ottawa, Ontario K1A 0R6, Canada.

Publisher's Note Springer Nature remains neutral with regard to jurisdictional claims in published maps and institutional affiliations.

The original article can be found online at https://doi.org/10.1007/ s12541-020-00338-4.

Wan-Ho Cho

chowanho@kriss.re.kr

1 Division of Physical Metrology, Korea Research Institute of Standards and Science, 267 Gajeong-ro, Yuseong-gu, Daejeon 34113, Korea

2 National Research Council Canada, 1200 Montreal Road, Building M36 - Office 1016, Ottawa, ON K1A 0R6, Canada

3 Department of Electronic Engineering, Cheongju University, 298 Daesung-ro, Cheongju 28503, Korea

4 Aichi University of Technology, Manori-50-2 Nishihasamacho, Gamagori, Aichi 443-0047, Japan

5 National Institute of Information and Communications Technology, 2-1-3, Katahira, Aoba, Sendai-city, Miyagi 980-0812, Japan 